\title{
Approches de l'objet enseigné. Quelques prolégomènes à une recherche didactique et illustration par de premiers résultats
}

\section{Christophe Ronveaux et Bernard Schneuwly}

\section{CpenEdition}

\section{Journals}

Édition électronique

URL : http://journals.openedition.org/educationdidactique/77

DOI : $10.4000 /$ educationdidactique. 77

ISBN : 978-2-7535-1614-4

ISSN : $2111-4838$

Éditeur

Presses universitaires de Rennes

\section{Édition imprimée}

Date de publication : 1 avril 2007

Pagination : 55-72

ISBN : 978-2-7535-0437-8

ISSN : 1956-3485

\section{Référence électronique}

Christophe Ronveaux et Bernard Schneuwly, « Approches de l'objet enseigné. Quelques prolégomènes à une recherche didactique et illustration par de premiers résultats », Éducation et didactique [En ligne], 1-1 | avril 2007, mis en ligne le 01 avril 2009, consulté le 08 décembre 2020. URL : http://

journals.openedition.org/educationdidactique/77 ; DOI : https://doi.org/10.4000/educationdidactique. 77 


\title{
APPROCHES DE L'OBJET ENSEIGNÉ QUELQUES PROLÉGOMÈNES À UNE RECHERCHE DIDACTIQUE ET ILLUSTRATION PAR DE PREMIERS RÉSULTATS
}

\author{
Christophe Ronveaux et Bernard Schneuwly, FAPSE, Université de Genève
}

\begin{abstract}
Résumé : la contribution comprend trois parties. Dans la première, nous fondons théoriquement l'idée de faire de l'objet enseigné en classe de français un objet de recherche didactique, dans le cadre de la théorie de la transposition didactique. L'unité d'analyse « séquence d'enseignement» nous sert de point de vue méthodologique pour aborder cet objet. Dans la deuxième, nous présentons une méthode empirique pour analyser l'objet enseigné. Elle consiste d'une part en un recueil de données où l'objet d'enseignement est au centre ; et d'autre part en une analyse prenant trois foci d'analyse : la séquence d'enseignement comme un tout ; quatre gestes fondamentaux d'enseignement; les interactions entre les différents domaines de la discipline français. Dans la troisième partie, nous illustrons les deux parties précédentes par une analyse, appartenant au premier focus, de 17 séquences d'enseignement du texte d'opinion. Nous montrons qu'on peut distinguer trois familles de suites d'activités qui permettent de classer les 17 séquences. Il s'agit de familles dont on peut supposer qu'elles circonscrivent les possibles de l'action des enseignants dans la construction de l'objet d'enseignement « texte d'opinion».
\end{abstract}

Mots clés : transposition didactique ; forme scolaire ; chaines de tâches ; séquence d'enseignement ; usage des textes et littérature

\section{Christophe Ronveaux E Bernard Schneuwly}

Il ne saurait être question, dans le présent texte, d'exposer en détail l'ensemble des concepts nécessaires pour approcher l'un des objets centraux de la didactique, le savoir enseigné, l'objet enseigné. Quelques brefs points de repère devront suffire. Nous définissons le didactique comme une construction socio-historique liée à l'avènement de l'école que nous attribuons à un contexte où croit la division entre travail manuel et intellectuel. Il s'agit d'un espace social spécialisé et institutionnalisé dans lequel quelqu'un enseigne quelque chose, prédéfini par l'institution - un objet d'enseignement -, à d'autres qui apprennent selon une logique prédéfinie par l'enseignement et qui déploient donc une forme spécifique d'apprentissage. Le processus de transposition est constitutif du didactique. Il résulte d'au moins deux caractéristiques de ce dernier : la nécessaire modélisation minimale des objets d'enseignement pour devenir enseignables et le changement de fonction des savoirs qui deviennent objets à enseigner et qui entrent dans le processus d'enseignement et se transforment en objets enseignés. Les effets de ces déterminants sur l'objet sont décrits dans de nombreux travaux sur la transposition didactique, qui cependant sont en grande partie consacrés à ce qui est en général désigné par le terme de « transposition externe ». Ils montrent l'extrême complexité de ses nombreux déterminants et du processus lui-même. Et pourtant, la saisie de la transposition interne résultant en un objet enseigné semble encore plus redoutable. Peu de travaux s'attellent à sa description; et encore moins, à notre connaissance, essayent de définir une démarche systématique, méthodologiquement armée, pour tenter de cerner cet objet nécessairement instable, fuyant, variable, insaisissable et d'une certaine manière inexistant en dehors de sa toujours éphémère construction dans l'interaction didactique. Sa description ne peut dès lors qu'être à son tour «cernement " par des voies multiples. C'est précisément une démarche de ce type que nous esquissons dans les lignes qui suivent en l'illustrant par une analyse de l'objet enseigné "production d'un texte argumentatif $»$ des classes de $8^{\mathrm{e}}$ Genevoise (5 $5^{\mathrm{e}}$ Française). 


\section{Objet enseigné et transposition didactique}

La transposition interne, à savoir celle passant de l'objet à enseigner à l'objet d'enseignement et enseigné, constitue la mise en texte du savoir en fonction de contraintes d'avancement dans le temps et de redéfinition continuelle des places. L'objet à enseigner devient un objet d'enseignement - nous nous référons ici à Chevallard (1991) -; il doit apparaître comme un objet à deux faces, à la fois nouveau pour être objet d'enseignement et enjeu d'apprentissage, et en même temps ancien, pour pouvoir être relié à ce qui est déjà là, déjà connu d'une certaine manière. Cette contradiction entre nouveau et ancien constitue le véritable moteur de la transposition didactique au niveau interne; il en gouverne le déroulement. La gestion du rapport entre nouveau et ancien définit d'une part la topogénèse, dans la mesure où le nouveau garantit à chaque moment l'hégémonie de l'enseignant dans le rapport au savoir. Sa gestion définit aussi la chronogénèse du savoir, dans la mesure où l'enseignant sait toujours en avance ce qui sera encore à faire et introduit le nouveau à enseigner en fonction de l'ancien, supposé appris. Notons cependant que le temps de l'enseignement - comme le montre Vygotski (1934/1985) à travers le concept de zone de proche développement - n'est jamais le temps de l'apprentissage. Il y a d'une part un temps fictif de l'enseignement, celui où l'on fait comme si l'élève suivait pas à pas un enseignement qui décompose les objets pour les rendre accessibles. Cette fiction est nécessaire pour donner accès, pour construire avec les élèves ces objets. D'autre part, l'élève, tout en suivant cette fiction, en utilisant les ingrédients donnés, en s'immergeant dans les situations proposées, bref en apprenant, construit lui-même les capacités liées aux savoirs, selon une logique qui n'est certainement pas celle du pas à pas de l'enseignement, mais qui procède par restructuration subite de pans entiers de son fonctionnement à certains moments. L'élève doit faire ce qu'il ne sait pas (encore) faire; les exigences sont au-delà de ses possibilités actuelles et pourtant il peut y répondre, au moins partiellement, grâce à la mise en scène didactique : décomposition des systèmes, simplification et graduation des situations, régulation de l'apprentissage par diverses formes d'évaluation. Ceci pose des problèmes complexes de prise en compte de l'ancien, préconstruit, supposé connu, et des modalités de reconstruction de l'ancien par des restructu- rations soudaines qui ne suivent pas nécessairement - justement - le temps de l'enseignement, qui doit dès lors sans cesse rappeler et faire comme si l'avancement se faisait selon une progression cumulative et régulière.

C'est le résultat de ce processus - objet à enseigner qui devient objet enseigné - qui est au centre de notre recherche. Il doit être défini de deux points de vue, interne et externe :

- Produit continuellement construit et transformé, comme nous venons de le voir, à travers la chronoet topogénèse, l'objet enseigné apparaît, du point de vue interne, comme une constante transformation de significations par découpage, par ajout et par reconfiguration du déjà là. Ce qui est au centre de ce regard est l'introduction du nouveau par rapport à l'ancien, le tissage continuel de significations nouvelles par transformation et enrichissement de significations; le regard ici est génétique; et le produit, l'objet enseigné, est le résultat de la genèse.

- Du point de vue externe, l'objet enseigné peut également être considéré comme le produit d'un processus ayant une certaine structure et qui définit d'une part son empan, d'autre part les caractéristiques générales selon lesquelles il est construit. C'est la structure de ce processus qu'il s'agit maintenant d'analyser plus en détail.

\section{La séquence d'enseignement comme unité d'analyse : hiérarchie et séquentialité du travail sur les contenus}

La transposition interne, nous venons de le voir, porte sur des savoirs ou objets à enseigner. Son analyse présuppose de définir une unité d'analyse qui correspond à un objet à enseigner. Qu'est-ce qu'au fait un « objet à enseigner » ? Quelles sont ses limites? D'une certaine manière, ce problème ne peut pas être résolu : tout objet à enseigner fait toujours partie d'un autre objet à enseigner qui l'englobe. Ainsi la subordonnée relative fait partie de la subordonnée et celle-ci de la phrase, et ainsi de suite. Inversement, un objet à enseigner est en général décomposable en unités plus petites qui sont également des objets à enseigner, comme par exemple la subordonnée introduite par qui ou par dont, ou la transformation de la phrase P par son enchâssement dans une autre phrase. Il paraît néanmoins possible - et il est méthodologiquement nécessaire - de découper dans le flux des objets enseignés à travers la suite 
ininterrompue des leçons - nous désignons ainsi les unités administratives qui structurent bon gré mal gré la temporalité matérielle de l'école ${ }^{1}$ - des unités relativement bien circonscrites, comprenant diverses activités articulées entre elles, organisées pour l'enseignement d'un objet à enseigner considéré comme un tout, avec un début et une fin délimités. Il n'est pas rare d'utiliser à ce propos, à l'école, la métaphore d'un nouveau chapitre qui s'ouvre ou qu'on reprend. Lobjet d'enseignement ainsi délimité a une certaine envergure, couvre en général plusieurs leçons et inclut plusieurs dimensions. Un tel objet d'enseignement qui structure le flux continu de l'enseignement donne lieu à une séquence d'enseignement, à savoir à un ensemble de leçons organisé systématiquement pour l'étudier comme tel, et du point de vue de ses différentes dimensions à prendre en considération; l'objet est déterminé en amont par les plans d'étude et/ou les manuels, et plus largement par l'institution. L'autonomie d'une séquence d'enseignement par rapport au reste de l'enseignement est relative, nous venons de le dire : en général l'objet a déjà été traité et va encore l'être; il peut s'insérer dans d'autres unités; il peut s'étendre sur des périodicités très longues; il est en général traité de manière discontinue. Nous défendons cependant théoriquement l'idée que le découpage de l'objet à enseigner en composantes est, par principe, indépassable dans la forme scolaire, même s'il peut être aménagé. L'unité d'analyse « objet enseigné dans une séquence d'enseignement » nous parait constituer une unité constitutive de l'objet général de la didactique : les conditions de transmission de savoirs dans une institution spécialisée.

Toute séquence d'enseignement vise la construction de nouvelles connaissances par les élèves en rendant accessible un objet à apprendre. Elle a deux caractéristiques principales qui permettent de réaliser cette finalité : elle est construite hiérarchiquement et, comme le dit son nom, elle se présente comme une séquence organisée dans le temps. En effet, dans la séquence d'enseignement, l'objet d'enseignement est rendu présent et décomposé en unités plus petites que l'élève peut étudier - observer, analyser, exercer - comme autant de facettes de l'objet à s'approprier. Ces facettes, loin de constituer le tout de l'objet, sont définies par la tradition scolaire, consignée dans les textes de références, mais également, plus encore, elles sont transmises à travers des pratiques dans une tradition souvent séculaire. En même temps, chaque séquence constitue toujours aussi un choix concret de décomposition de l'objet par un enseignant donné dans une classe donnée. Cette décomposition de l'objet implique une mise en hiérarchie qui peut comporter plusieurs niveaux : l'objet luimême, certaines de ses dimensions, certains éléments de chacune de ses dimensions, etc. Cette hiérarchie se distribue séquentiellement dans le temps: un élément donné vient après ou avant l'autre. Elle peut se manifester en ouverture soit sur un démarrage de la séquence par un élément simple pour aller vers le complexe, soit par un démarrage de l'objet complexe pour aller vers sa décomposition, et éventuellement recomposition. Sous-jacente à la séquentialité il y a cependant très souvent l'idée d'une progression dans la construction de l'objet.

La structure nécessairement séquentielle et hiérarchique de la séquence d'enseignement est un effet de la forme scolaire et résulte du fait que l'apprentissage se réalise grâce à l'enseignement, dans des situations spécialement créées pour apprendre, dans un dispositif d'apprentissage progressif allant dans le sens d'une complexification et d'une spécialisation des savoirs construits. Du point de vue de la compréhension et de l'explication de la construction de l'objet enseigné, la séquence d'enseignement semble ainsi constituer l'unité d'analyse optimale dans la mesure où elle contient tous les éléments essentiels du processus de transposition interne: chronogénèse et topogénèse dans un processus hiérarchique de décomposition et de recomposition de l'objet, et séquentiel d'organisation d'une progression. Au-delà de la séquence hiérarchique, l'objet d'enseignement risque de devenir trop général et insaisissable, se confondant avec des parties entières de la discipline scolaire; en-deça, c'est l'organisation hiérarchique et peut-être même séquentielle au sens où nous venons de la définir qui risque de disparaître. Mais surtout, c'est à travers une séquence d'enseignement qu'un objet à enseigner est transformé en objet enseigné; la séquence d'enseignement est le lieu temporel et spatial où se construit dans l'interaction entre élèves et enseignant, ce qui constitue l'objet de notre enquête. C'est la raison principale d'en faire notre unité d'analyse.

La question que nous nous posons est d'abord, à un niveau général et abstrait, de connaître et de comprendre, comment l'objet à enseigner se transforme en objet enseigné. Cette question principale peut maintenant être spécifiée comme suit : quelles sont les contraintes, historiques, didactiques, institu- 
tionnelles, qui déterminent constance et variation de l'objet enseigné? Dans quelle mesure cette variation dépend des objets d'enseignement? Et quel est l'effet des réformes des trente années passées des plans d'études et moyens d'enseignement sur les objets enseignés : continuité, rupture, ou sédimentation?

Cette définition générale de la problématique implique une série de décisions méthodologiques qu'on peut définir comme suit :

1. Nous nous donnons comme unité d'analyse - nous venons de l'expliciter - le lieu institutionnel, borné par l'enseignant, à travers lequel s'opère pour l'essentiel le processus de transformation des objets d'enseignement : la séquence d'enseignement. Elle permet en même temps de délimiter l'empan de l'objet à enseigner.

2. Notre recherche porte sur un objet d'enseignement délimité, prévu dans le plan d'étude, dont il s'agit d'observer la transformation à travers une séquence d'enseignement. La définition de l'objet doit à la fois garantir une possibilité de comparaison pour le chercheur et laisser une latitude suffisamment grande de projection et d'interprétation de la part des enseignants. Autrement dit : le contrat entre chercheur et enseignant doit à la fois préciser un objet d'enseignement de manière suffisamment précise pour rendre des comparaisons possibles, et laisser à l'enseignant la plus grande liberté de choix pour l'enseignement de cet objet.

3. Il paraît intéressant d'observer la transformation du même objet d'enseignement dans le travail de plusieurs enseignants pour mieux déterminer les contraintes à travers les effets de constance et de variation.

4. Et d'observer les transformations opérées sur plusieurs objets à enseigner de manière comparative, les contraintes agissant sur le processus dépendant sans doute aussi de la nature même de l'objet.

Ces quatre éléments d'opérationnalisation délimitent a priori un objet d'enseignement, proposent une démarche comparative - entre enseignants travaillant le même objet, et entre objets - et définissent une unité d'analyse et d'observation dans le flux général de l'enseignement.

\section{Observer et analyser des séquences d'enseignement pour décrire l'objet enseigné}

\section{Recueil des données}

Ces éléments théoriques ont des conséquences concernant la méthode de recherche ${ }^{2}$. La première se situe au niveau du choix des objets. Notre empan d'observation a été défini par le choix d'un objet à enseigner prévu par le plan d'étude. Deux objets ont été retenus: d'une part, ce qu'on appelle, dans le cadre de l'argumentation et de la production écrite de textes, le texte d'opinion, représentant les textes argumentatifs, et d'autre part, dans le cadre de la grammaire de phrases, la proposition subordonnée relative ${ }^{3}$. Ces deux objets « partiels » se trouvent dans les plans d'études, et sont censés être enseignés dans des classes d'élèves de 14-15 ans. Le contrat avec les enseignants volontaires pour cette observation était qu'ils devaient enseigner tel ou tel objet. Tout cela, simple et clair sur le papier, s'est évidemment révélé beaucoup plus complexe dans la réalité, en particulier du point de vue de la durée : enseigner le texte d'opinion peut s'étendre sur l'année comme être concentré sur quelques jours. La subordonnée relative peut être simplement « revue » en une période, ou être incluse dans un travail beaucoup plus long d'analyse des propositions. Toutefois, dans le fonctionnement actuel de l'école, on peut déterminer des sortes de " chapitres », d'une durée variable mais avec un début et une fin, consacrés au texte d'opinion ou à la subordonnée relative. Et c'est cet empan-là que nous avons décidé d'observer, empan pouvant aller d'une période administrative à une quinzaine de périodes. Le recueil de données a donc été pensé en fonction de l'objet enseigné tel que défini plus haut.

Le contrat entre chercheur et enseignant a par conséquent été défini comme suit : l'enseignant s'engage à enseigner l'objet défini par le plan d'études, à un moment donné et à permettre au chercheur de venir enregistrer son enseignement. Aucune consigne quant à la durée, aux moyens d'enseignement à utiliser ou aux dimensions de l'objet à enseigner n'est donnée. Le chercheur insiste auprès de l'enseignant pour qu'il enseigne, dans la mesure du possible, « comme d'habitude ». Des entretiens précèdent et suivent les enregistrements. Lors de regroupements, les chercheurs rendent compte de leurs premières analyses et discutent avec les enseignants de leurs différentes manières d'aborder les mêmes objets d'enseignement. 
Il a fallu également prendre des décisions quant à l'enregistrement en classe lui-même : quel type de caméra, doubler avec un mini-disc, comment placer les microphones, où placer tout cela, focaliser sur qui (Hall, 2000; Stigler, Gallimore \& Hiebert, 2001) ? De fait, ces points font partie de l'objet de la recherche, le recueil des données constituant déjà une interprétation de la réalité, et notre propos visant l'observation du travail de l'enseignant. Pour le recueil des données, il a fallu nous limiter à une moyenne de 5 périodes transcrites par enseignant, en général le début et la fin de la séquence didactique (en excluant en général la période consacrée à l'évaluation), et trois ou quatre périodes intermédiaires que l'enseignant estimait importantes. Nous avons ainsi la transcription de 13 enseignants pour la subordonnée relative et de 17 enseignants pour le texte d'opinion. Soit sur les 30 classes observées, 150 leçons, 3000 pages de transcription.

\section{Traitement des données et première analyse}

La transcription étant elle-même une théorie (Ochs, 1979), il a fallu également décider comment transcrire et que transcrire : non seulement ce qui était dit, avec des repères chronologiques, mais comment, les gestes, la prosodie, l'utilisation des moyens d'enseignement (manuels, feuilles d'exercices, textes ou extraits de textes à lire, etc.), du tableau noir, sans compter le recueil de ce que l'enseignant utilisait matériellement (photocopies, transparents, etc.), tout cela annexé à la transcription. La définition de l'objet enseigné, avec cette centration sur le travail de l'enseignant, a donc conditionné très étroitement toutes les décisions méthodologiques prises au niveau de l'observation et de la transcription.

L'analyse de l'objet enseigné s'est basée sur ces 3000 pages de transcription (relativement) détaillée, et a présenté deux étapes bien distinctes. La première a consisté à élaborer un synopsis, permettant d'avoir une vue d'ensemble de la séquence, les périodes se succédant sur un tableau. Le synopsis, au sens étymologique de "voir ensemble », est un outil heuristique qui permet de comprendre comment, à chaque instant (de l'analyse), l'objet enseigné est en lien avec la totalité de la séquence; on ne peut interpréter tel instant de telle période portant sur tel élément de l'objet enseigné qu'en lien avec la totalité de cet objet comme connaissance - de la subordonnée relative ou du texte argumentatif ou d'opinion ${ }^{4}$. Cette première étape consiste donc à réduire les données - également leur élémentarisation! - pour les rendre "lisibles » et maîtrisables. Cette étape de réduction permet également de faire émerger le déroulement séquentiel de la séquence didactique, avec des niveaux hiérarchiques (n, nn, nnn, nnnn) correspondant à la suite des milieux, tâches et dispositifs didactiques mis en œuvre par l'enseignant pour rendre l'objet présent et pour en faire travailler des aspects. Nous sommes donc là au niveau de la séquentialité de l'objet.

En nous inspirant librement des théories de la communication multimodale (Jewitt \& Kress, 2003), nous avons montré ailleurs (Dolz, Ronveaux \& Schneuwly, 2006; Ronveaux \& Simon, en préparation) que les indicateurs d'ordres sémiotiques distincts permettent d'identifier dans le flux des interactions didactiques des blocs hiérarchisés. Aux niveaux hiérarchiques 'n' et 'n.n', nous avons regroupé les activités scolaires que devaient effectuer les élèves, ou les milieux dans lesquels ils étaient mis pour l'étude, et dans les niveaux 'n.n.n' et 'n.n.n.n', le détail des activités. Chaque niveau et chaque moment sont décrits par un résumé narrativisé, en mentionnant l'emplacement dans la transcription, la forme du travail scolaire, et les supports utilisés (moyen d'enseignement ou document personnel). Toutefois, il faut reconnaître que pour ces regroupements hiérarchisés une part importante est laissée à l'inférence et à l'interprétation d'indicateurs relevant de modalités multiples, depuis le discours de l'enseignant jusqu'à l'organisation matérielle de la classe. On peut parler du passage d'une sémiotique à une autre (Rastier, 2001). Le synopsis permet donc de condenser en une unité appréhendable sur un tableau les transcriptions des séquences d'enseignement afin de les rendre analysables et comparables, et de mettre en évidence la structure hiérarchique et séquentielle (niveaux supérieurs, description des activités scolaires) de chaque séquence d'enseignement. Le synopsis a donc été notre première étape, comme outil méthodologique qui nous permet de saisir en première lecture et en première analyse le tout de la séquence.

\section{Trois foci d'analyse 5}

La tentative de saisir une réalité aussi fuyante, changeante, multiforme, partiellement virtuelle que l'objet enseigné nécessite la mise en œuvre d'une méthode qui capte le phénomène de plusieurs points de vue, qui l'observe à des distances différentes, qui 
le dissèque avec des instruments variés ${ }^{6}$. Et c'est la mise en commun des résultats obtenus à travers la mise en ouvre de modalités complémentaires de saisie des données qui permettra éventuellement de tirer quelques conclusions plus générales concernant les manières de construire les deux savoirs constituant l'objet de notre recherche (subordonnée relative et texte argumentatif).

Pour atteindre cet objectif, nous avons dans un premier temps défini trois foci saisissant l'objet avec un degré de précision, un grain de résolution plus ou moins précis, ceci mettant en évidence, par définition, des facettes différentes de l'objet. Bien sûr, pour chaque focus, les points de vue de saisie sont également variés, nous y reviendrons.

\section{Premier focus}

Le premier focus porte sur les séquences comme un tout, ou comme un ensemble de parties structurées. Il est donc ici centré sur la manière des enseignants de découper l'objet pour le rendre accessible, et sur la manière d'ordonner les éléments les uns après les autres. Le but visé est l'établissement d'une première typologie de séquences et de démarches de construction de l'objet enseigné. Dans ce premier focus, nous adoptons deux démarches :

- Analyse du niveau n et n.n, pour produire une description permettant d'obtenir une première description des contenus et démarches dominantes et de leur trame prototypique.

- Analyse au niveau n.n.n du synopsis, pour obtenir une première description d'ensemble des activités scolaires mises en œuvre et de leur enchaînement.

Nous illustrerons ce focus d'analyse dans la deuxième partie à propos des séquences portant sur le texte argumentatif.

\section{Deuxième focus}

Nous l'avons vu plus haut : construire un objet d'enseignement implique une série de gestes professionnels fondamentaux qui rendent présent l'objet en classe et qui pointent certaines de ses dimensions essentielles. Nous utilisons le terme ici à un niveau plus général, faisant référence à un autre usage possible du terme que traduit bien le terme " gestus». Le terme introduit une première fois par Brecht dans son essai du Petit Organon désigne cet ensemble de traits déterminant un type humain à partir desquels l'acteur de théâtre peut construire son personnage.

«Sous le terme gestus, il faut entendre un ensemble de gestes, de jeux de physionomie et (le plus souvent) de déclarations faites par une ou plusieurs personnes à l'adresse d'une ou de plusieurs autres. Une personne qui vend un poisson montre entre autre le gestus de la vente. Un homme qui rédige son testament, une femme qui séduit un homme, un policier qui passe à tabac, un homme qui verse leur paye à dix autres : il y a là toujours un gestus social. [...] Des paroles, des gestes peuvent être remplacés par d'autres paroles, d'autres gestes, sans que le gestus s'en trouve modifié. » (Brecht, 2000/1940, p. 892s)

De même, porteurs de significations, ces gestes fondamentaux s'intègrent dans le système social complexe de l'activité enseignante qui est régi par des règles et des codes conventionnels, stabilisés par des pratiques séculaires constitutives de la culture scolaire. Dégager ces gestes de la profession enseignante suppose donc une clarification des situations et des activités scolaires dans lesquelles ils prennent place, ainsi que des outils et des démarches permettant leur mise en acte.

Nous utilisons cette particularité du travail enseignant pour analyser les séquences d'enseignement du point de vue de ces gestes professionnels fondamentaux effectués par tous les enseignants pour enseigner les deux objets analysés, chacun de ces gestes étant considéré comme révélateur de l'objet enseigné. Ces analyses permettent, dans un deuxième temps, de revenir sur les séquences et les enseignants et de les caractériser à partir de typologies élaborées à partir de l'ensemble des gestes repérés. Le principe d'analyse de ce deuxième focus est donc de repérer dans les séquences les gestes professionnels et d'analyser les dimensions de l'objet sur lesquelles ils portent. Nos analyses reconstruisent l'objet enseigné en partant de quatre gestes fondamentaux (nous nous inspirons ici de la théorisation proposée par Sensevy, Mercier, Schubauer-Leoni, Ligozat, \& Perrot, 2005).

- Mettre en ouvre des dispositifs didactiques : dans le cours de l'enseignement, l'élémentarisation de l'objet sous des formes diverses que nous avons regroupées sous le terme générique de 'dispositif didactique'. Analyser les dispositifs didactiques revient dès lors à s'intéresser à la manière dont l'objet d'étude est rendu présent à travers les différents supports mobilisés par l'enseignant, plus particulièrement à travers la consigne et le matériau didactique qu'elle met en œuvre. 
- Réguler : la régulation du processus d'enseignement/apprentissage se fait à différents niveaux et peut s'observer de différents points de vue. Dans notre travail, nous observons la régulation par l'enseignant à deux niveaux : la régulation interne et la régulation locale. Par régulation interne, nous entendons des démarches outillées servant à obtenir des informations sur l'état des connaissances des élèves. Les régulations locales opèrent durant les activités scolaires, à la faveur d'un échange avec l'élève. Leur analyse à l'intérieur d'une séquence d'enseignement permet de pointer des interactions dont on peut faire l'hypothèse qu'elles conduisent à la construction de l'objet enseigné. Nous menons une troisième analyse de la régulation. En effet, nous analysons comment l'enseignement/apprentissage est régulé quand un obstacle est repéré auprès d'un ou plusieurs élèves ou quand un élève amène des éléments de contenus non prévus.

- Institutionnaliser : le geste de l'institutionnalisation fixe, à un moment donné de la séquence didactique, des éléments de l'objet d'enseignement comme devant être connu sous une forme conventionnelle, l'enseignant définissant la forme et le contenu de cet objet. Il s'agit donc d'un geste particulièrement intéressant pour connaître l'objet enseigné en classe.

- Construire la mémoire didactique : l'analyse de la construction de la mémoire didactique passe par une mise en relief des moments où la mémoire est convoquée, aussi bien dans sa fonction de rappel (référence à la mémoire de la classe, de l'élève, des activités scolaires et/ou de l'objet enseigné) que dans sa fonction d'appel et d'anticipation (notamment à la mise en mémoire).

\section{Troisième focus}

Le troisième focus dépasse l'objet enseigné en tant que tel et pose la question de savoir comment cet objet est construit en s'appuyant sur d'autres provenant d'autres domaines de l'enseignement du français. En effet, enseigner le français se construit historiquement comme un processus circulaire entre différents domaines, notamment grammaire, lecture, écriture (Schneuwly, 2007 ; pour une vision actuelle de la didactique du français, voir Falardeau, Fisher, Simard et Sorin, 2007). Nous essayons ainsi de travailler ce qui constitue profondément la discipline du français, à savoir, comme nous l'avons dit plus haut, sa structure en triptyque. Pour atteindre cet objectif, nous procédons à deux analyses : nous explorons d'une part l'usage de notions grammaticales dans le travail sur le texte; et nous observons l'utilisation de textes (littéraires) dans la construction de la notion de subordonnée relative et dans la construction de la capacité de produire des textes argumentatifs. Permettre cette circulation des objets entre différents domaines du français nous semble constituer ce qu'on pourrait appeler des gestes spécifiques du professeur du français.

Les trois foci se complètent: le premier met au centre les séquences didactiques dont l'empan est déterminé précisément par le travail sur l'objet enseigné tel que défini dans le plan d'étude et dans le contrat de recherche. Le deuxième porte sur les gestes fondamentaux, unités constitutives des séquences, avec un grain plus fin de l'analyse permettant de saisir plus en détail des éléments constituant l'objet enseigné. Le troisième est en quelque sorte double - on pourrait parler de regard bifocal : il met sous la loupe les lieux de croisement entre domaine du français; il saisit l'objet aux endroits où il se trouve en intersection avec d'autres objets, le montrant comme nécessairement composite. Le focus porte donc sur des éléments bien circonscrits de la séquence et en même temps en révèle ses lieux de passages avec les autres dimensions de la discipline français.

\section{Une illustration : une analyse des séquences d'enseignement portant sur le texte argumentatif}

\section{Comprendre la logique d'une séquence}

Pour illustrer la démarche d'analyse des séquences, concentrons notre attention sur une partie de notre collection, celle qui comprend les 17 séquences d'enseignement portant sur le texte argumentatif ${ }^{7}$. Saisir la séquence comme un tout présuppose que l'on reconnaisse à ce tout un ordonnancement. La question du lien sur lequel est construit cet ordonnancement se décline en terme de logique, la question chronologique étant un des effets de cette logique. Pour commencer notre analyse, il faut parier sur le fait que la parcellisation ou l'atomisation des savoirs, tant de fois décriée, a un sens, une direction qui oriente l'objet à enseigner vers l'objet effectivement enseigné. Comment conduire cette analyse? Lintelligibilité de ce tout se comprend entre deux bornes, en amont et en aval de l'objet d'enseignement. Il faut d'abord admettre que dans le flux de ce qui s'enseigne il y a une entrée en matière et une limite en aval. Ce 
début et cette fin doivent se comprendre comme une rupture dans la succession des objets et sous-objets travaillés. Dans le présent article, nous n'avons pas examiné ces ruptures externes à la séquence. Cellesci sont préfigurées par le contrat de recherche et les objets d'enseignement choisis en fonction des plans d'études (cf. supra). Nous n'avons considéré que les ruptures internes de la séquence : en amont, ce qui distingue la mise en présence de l'objet d'enseignement de la suite de la séquence, et en aval, ce qui distingue la ou les activités successives internes à la séquence de celles qui terminent la séquence et la finalisent, en somme. Une fois identifiés le début et la fin des séquences, nous avons conduit notre analyse sur les liens qui structurent les suites d'activité internes aux séquences. Ces deux premières étapes de l'analyse ont été portées sur trois séquences de la collection, puis sur l'ensemble des séquences (désormais, les séquences seront désignées par TO01, TO02, etc. dans la suite du texte).

Parmi tous les outils à disposition exposés cidessus pour cerner l'objet d'enseignement, nous avons utilisé le synopsis et avons effectué des allers et retours entre ce dernier et les transcriptions pour préciser certaines activités. Un mot encore sur les tableaux synoptiques reproduits en annexe : ils présentent une version simplifiée des synopsis et « résument » en quelque sorte les séquences d'enseignement TO16, TO07 et TO05 dont les durées varient respectivement entre 7, 6 et 9 périodes. Pour les besoins de la démonstration, le choix de ces trois séquences a été dicté après l'analyse de l'ensemble de la collection; elles nous ont semblé représentatives de la manière dont se structurent des séquences d'enseignement.

\section{Les ouvertures et les fermetures}

Que nous apprend un coup d'œil rapide sur les ouvertures des trois séquences représentées par les tableaux en annexe? La séquence TO16 (cf. la figure 4 en annexe) s'ouvre sur une activité de définition; cette entrée en matière concerne un objet du monde, l'activité d'argumenter. L'examen plus détaillé de l'activité proposée par l'enseignant et l'observation des retours qu'il porte sur les formulations des élèves confirment cette activité de référence. L'enjeu de l'activité est bien pour l'enseignant de satisfaire aux conditions ontologiques d'une réalité du monde qu'il s'agit de définir par un travail de formulation mis en correspondance avec la définition d'un dictionnaire. La définition dictée par l'enseignant à la fin de l'activité est symptomatique de l'abstraction de cette réalité du monde («Argumenter, c'est donner et défendre une opinion en la justifiant grâce à des exemples, à vos connaissances ou à des informations prouvées. C'est aussi essayer de convaincre quelqu'un de quelque chose »). Par contraste, la séquence TO07 (cf. la figure 5 en annexe) s'ouvre sur une activité de définition d'un objet textuel, la pétition, l'objectif étant de déterminer ses conditions de production (quel est son signataire? Son destinataire? Ses enjeux d'influence? Ses objectifs? Etc.). C'est un objet de la langue qui est mis en définition.

La séquence TO05 (cf. la figure 6 en annexe) est très différente des deux précédentes. Son ouverture est récursive et déterminée par le texte littéraire. Organisée sur la mise en présence de trois fables de La Fontaine, elle s'ouvre trois fois de la même manière : (1) lecture à voix haute du texte, (2) compréhension globale du récit et (3) explicitation des mots du texte incompris. L'objectif est de mettre à la disposition des élèves l'ensemble du texte, de s'assurer de leur compréhension du récit, et de rapidement passer au cœur de la séquence, à savoir le travail d'explicitation sémantique des situations argumentatives représentées par les fables. Cette ouverture en trois temps concerne en effet le texte littéraire et ses modalités de traitement «classiques » par lesquelles il s'agit d'abord de le découvrir linéairement comme un tout, à la fois dans ses dimensions matérielles, graphique et sonore, et dans sa dimension thématique (pour autant que le récit soit un thème, dans ce cas-ci) avant de lancer les élèves dans une activité d'analyse ou de commentaire. Tandis que ces activités d'ouverture ne servent pas directement l'objet argumentatif, elles donnent à voir l'approche littéraire de l'objet d'enseignement. Par celles-ci, nous indiquons que l'entrée de la séquence passe par le texte, depuis sa manifestation linéaire jusqu'au récit qui le constitue comme un tout, comme un préalable du travail sur l'argumentation.

Qu'en est-il des fermetures des séquences? Toutes les trois se terminent par un exercice de production de texte. Les genres de texte varient, et partant la nature de l'exercice. Le texte attendu en TO16 est une dissertation, le texte attendu en TO07 une pétition, le texte attendu en TO05 la réécriture de la morale de la fable vue en classe. La première séquence aboutit à la production de texte; les deux autres séquences se terminent par ce qu'il est convenu d'appeler des écritures d'invention, au sens défini par Daunay (2004). Quelle est la logique d'ordonnancement qui 
préside au passage de ces deux limites? Quels sont les liens qui assurent le passage d'une définition à l'écriture d'une dissertation, de l'identification d'un objet textuel à l'écriture d'une pétition, de la compréhension d'une fable à la réécriture d'une morale?

\section{Les liens internes des chaînes d'activité}

Les liens qui structurent la suite d'activité de TO16 sont construits sur l'expansion d'une idée de départ. La progression se réalise en deux temps. Premier temps, l'expansion de l'idée (du "thème ", de la "thèse ", de la «théorie», selon les termes de l'enseignant) en arguments opposés, confectionnés « en vrac », l'unité de base étant le paragraphe. Deuxième temps, l'articulation des arguments en mouvements argumentatifs, l'unité de travail étant le « corps du texte»ou le « développement » et les connecteurs assurant les «transitions logiques». Chacune de ces étapes est organisée en alternance par des conseils listés sans hiérarchie (l'ensemble de ces conseils est regroupé en un «memento » qui constitue la théorie) et des exercices pratiques de rédaction. Au-delà des particularités méthodologiques de cette séquence, nous représentons cette progression par le schéma de la figure .

Les liens qui structurent la suite d'activité de TO07 sont doubles : à un premier niveau, encadrant, ils portent sur les activités de production en amont (la production initiale) et en aval (la production finale) de la séquence, chacune de ces activités de production étant pourvue d'un statut propre; à un deuxième niveau, subordonné au précédent, les liens portent sur la parcellisation des éléments constituants de la pétition (successivement, la demande, la justification de la demande et la mise en page de la pétition). La pétition est abordée d'emblée dans sa complexité de tout textuel à partir de ses conditions de production. Nous représentons cette progression par le schéma de la figure 2 (cf. page suivante).

Les liens qui structurent la suite d'activité de TO05 se portent à un niveau plus englobant, celui de l'entité textuelle. Chacun des textes littéraires est travaillé pour lui-même. Même si la visée est bien de pouvoir rédiger en finale de la séquence ce qui est considéré comme un texte argumentatif, chaque texte concentre une petite chaîne d'activités qui peut se réaliser indépendamment des autres. Ces chaînes conduisent de la découverte du texte vers la rédaction d'une morale portant sur le thème identifié dans la fable travaillée. La troisième chaîne comprend une comparaison de la fable de La Fontaine avec les fables sources d'Esope et de Phèdre, ce qui l'apparenterait à une démarche herméneutique de type philologique. Nous représentons cette progression par le schéma de la figure 3 (cf. page suivante).

Ces schématisations des suites d'activité des séquences TO16, TO07 et TO05 rendent compte des priorités choisies par trois enseignants. Ces schémas ne valent que pour les trois occurrences singulières attestées par l'empirie. Mais c'est la comparaison de ces occurrences avec l'ensemble de la collection qui permettra de donner sens aux séquences d'enseignement et de donner à voir, par la récursivité d'une structure, des pratiques sédimentées.
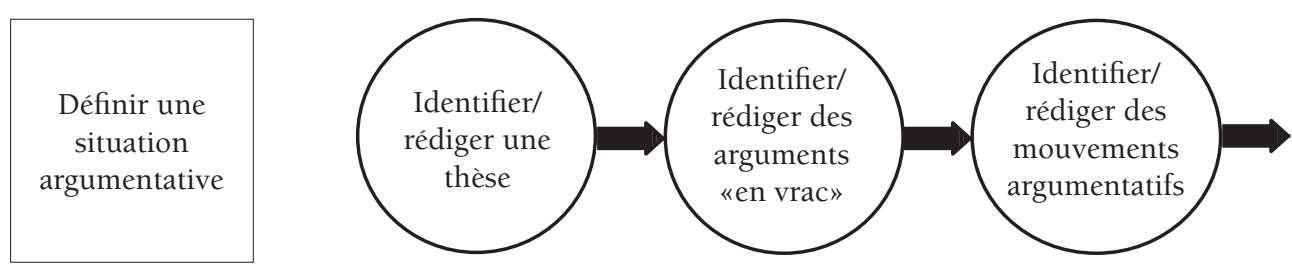

Écrire des textes d'idée (dissertation)

Figure 1. Schéma de la suite d'activités de TO16. 


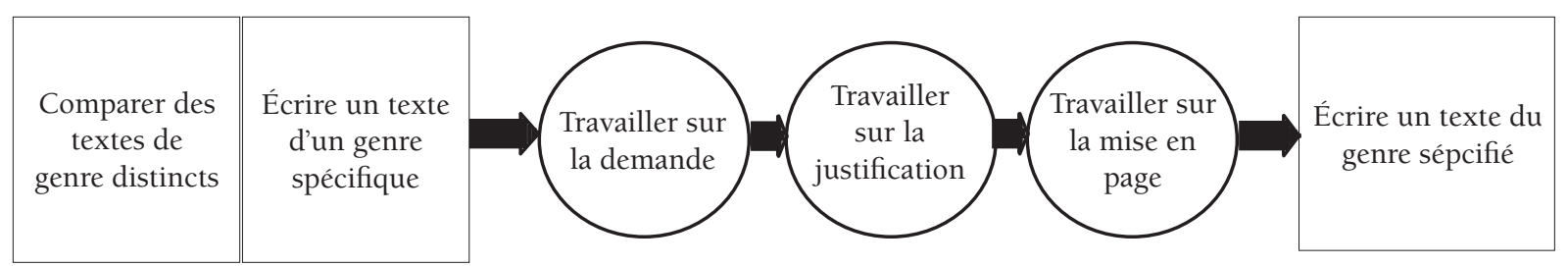

Figure 2. Schéma de la suite d'activités de TO07.

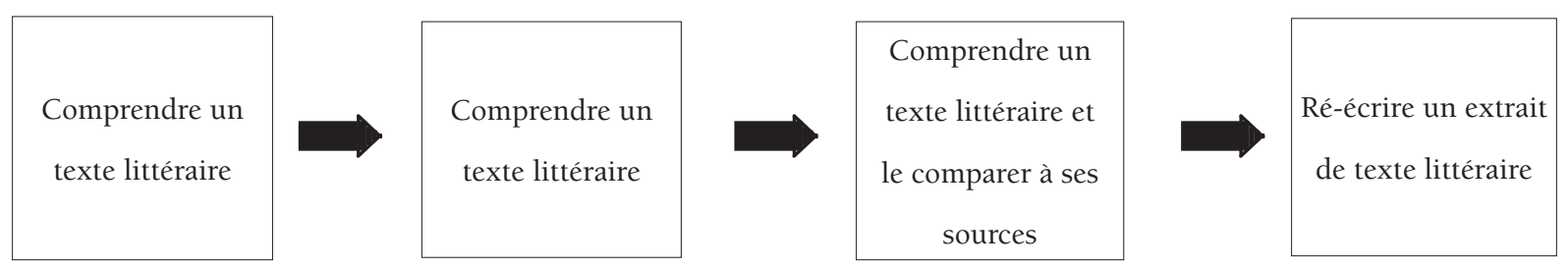

Figure 3. Schéma de la suite d'activités de TO05.

\section{Trois logiques d'organisation séquentielle et hiérarchique}

La comparaison des trois séquences ci-dessus nous donne à voir des logiques d'ensemble très différentes que nous pouvons tenter de généraliser à l'ensemble de la collection des séquences TO. Laissons de côté les séquences dont nous ne pouvons pas établir les débuts de manière assurée, à savoir TO01, TO03, TO12. Pour TO06, la mémoire didactique sollicitée par l'enseignante au début de notre captation donne suffisamment d'éléments pour reconstituer les composantes de l'objet d'enseignement travaillées. Quant à TO12, qui porte sur la mise en espace d'une réécriture du Procès de Montserrat d'Emmanuel Roblès, elle présuppose un travail de compréhension sur le récit et la constellation des personnages qui l'apparente à la séquence TO05, dont nous avons détaillé la description supra. En fonction des ouvertures et des fermetures, en fonction des liens qui assurent la succession des activités, on peut classer l'ensemble des séquences de notre collection en trois groupes.

Les séquences du premier groupe sont de loin les plus nombreuses (TO04, 06, 08, 09, 10, 14, 15, 16, 17) et relève d'une logique représentationnaliste. Elles partent de la représentation de la situation argumentative et aboutissent à l'écriture d'un texte d'idée ( "dissertation», " composition d'idées») ou d'une partie (introduction, conclusion ou arguments). Bien que les séquences TO04 et TO06 annoncent travailler sur une définition du « texte argumentatif , selon les termes utilisés par les enseignants, l'examen détaillé des tâches d'ouverture des séquences TO04 et TO06 révèle que c'est davantage la situation argumentative qui est définie, et cela indépendamment de son support textuel, et indistinctement à l'écrit ou à l'oral. Il s'agit bien pour toutes ces séquences de stabiliser la représentation d'un objet du monde, l'argumentation, qui met en présence au moins deux protagonistes qui tiennent des propos antagonistes sur le monde (discutent, échangent, se confrontent).

Pour les séquences de ce premier groupe, la situation argumentative est pensée comme un objet du monde, abstraitement, philosophiquement, pourrait-on dire. Dans ces séquences, le texte est utilisé comme une ressource langagière parmi d'autres, comme le débat ou la discussion, sans spécification de genre. Pour parvenir à la dernière activité d'écriture, les élèves passent par une progression en trois étapes: (1) l'idée, incarnée dans une thèse ou le plus souvent dans un thème, est travaillée au niveau local de la proposition; (2) les arguments, expansions du thème, sont travaillés au niveau du paragraphe; (3) l'articulation des arguments est travaillée en mouvements argumentatifs à l'échelle du « corps du texte », entre l'introduction et la conclusion, au moyen des connecteurs.

Les suites de tâches des séquences du deuxième groupe s'organisent sur un modèle communicationnel où le texte occupe la première place. Ce dernier est analysé, décrit et produit comme une performance sémiotique qui relève d'un sujet situé, pourvu d'objectifs spécifiques et pratiques, et de pratiques sociales normées, les genres. Il s'agit de 
la lettre ouverte en TO02, de la pétition en TO07 et TO11, du texte de point de vue en TO08, de la note critique de lecture en TO13 et de la lettre de réclamation TO15 (rangée sous la rubrique « Argumentation épistolaire »). Certaines séquences (TO07, TO08, TO13) se revendiquent explicitement de la logique de la séquence didactique, telle qu'elle est définie par les moyens d'enseignement S'exprimer en français (Dolz, Noverraz \& Schneuwly, 2001/2002). Dans une grande homogénéité des enchaînements ${ }^{8}$, les séquences de ce groupe s'ouvrent toutes sur une identification des caractéristiques du genre visé. Cette identification se fait soit par comparaison avec des textes d'autres genres (poésie, récit, définition de dictionnaire pour TO04) ou de genres proches (pétitions, lettre de demande, lettre de réclamation pour TO11), soit par explicitation des conditions de production du texte travaillé (TO07, TO08, TO13 et TO15). Même si cette dernière séquence TO15 ne se revendique pas des moyens d'enseignement mentionnés, il est à remarquer que la contextualisation qui précède systématiquement la lecture des textes concerne les enjeux d'influence des différents protagonistes (le requérant et le destinataire) qu'implique une réclamation. Pour l'ensemble des séquences de ce groupe, les chaînes d'activités s'organisent à partir des conditions de production du texte, puis se poursuivent sur les composantes langagières caractéristiques du texte génériquement identifié.

Quant au troisième groupe, il se compose de deux séquences seulement, TO05 et TO12. Les suites de tâches de ces séquences s'organisent de manière récursive autour du texte d'auteur dont il s'agit de garantir la lettre. Chacun des textes est rendu présent par une lecture à voix haute des élèves ou de l'enseignant, puis travaillé dans la visée d'une réécriture. Dans le cas de TO12, cette réécriture concerne la mise en espace du procès de Montserrat. Précisons que quelques séquences appartenant aux deux autres groupes conduisent une suite d'activité sur des textes littéraires selon la structure décrite supra. Ces suites sont conduites selon la logique d'une analyse de texte, indépendamment de la logique d'ensemble de la séquence. Ce sont les conclusions de l'analyse qui servent l'ensemble. Dans deux cas (TO04, TO10), ces conclusions illustrent la situation argumentative, dans un autre (TO16) la notion d'argument, et dans la dernière (TO17) la thèse.
Apportons trois nuances à ce premier classement. La séquence TO04, tout d'abord, mérite un traitement singulier. Elle s'ouvre sur une activité de classement de textes de genres distincts; parmi ces textes, on compte un schéma technique, une entrée de dictionnaire, un sonnet, le début d'un roman de San Antonio, la fable de La Fontaine " Le Loup et L'Agneau ». Ces textes sont disposés de manière « sauvage » sur une feuille A3. Les extraits présentés sont coupés suivant une ligne irrégulière qui rend la lecture de l'extrait difficile. Les élèves sont invités à classer les «types de textes", selon les mots de l'enseignant, à expliciter leur contexte de production en fonction de leur but, et à reconnaître les textes argumentatifs. Malgré cette ouverture qui devrait l'associer aux séquences du deuxième groupe, les liens internes de la chaîne des tâches combinent les logiques herméneutique et représentationnaliste. Considérons les deux tâches de définition et de lecture qui suivent immédiatement. La tâche de définition relève de ce même souci de définir une situation argumentative générale que nous avons identifié dans les séquences du premier groupe. Lanalyse des diverses situations de communication aboutit de fait à une définition qui échappe à la variété des contextes explicités. Quant à la lecture de la fable «Le Loup et L'Agneau », le travail mené autour du texte littéraire conduit à une illustration de la définition formulée précédemment. C'est bien de propriétés ontologiques de l'argumentation qu'il est question dans ces deux tâches.

De même, la séquence TO08 est complexe à analyser. Alors qu'elle s'appuie explicitement sur les moyens d'enseignement de la "Commission romande des moyens d'enseignement " (Dolz, Noverra, Schneuwly, 2001/2001) et suit les modules d'enseignement proposés par le manuel (les supports utilisés sont extraits de la séquence sur « Le point de vue », S'exprimer en français, volume IV), la conduite des tâches dérive sur d'autres objets que ceux prévus par la séquence. Les interventions de l'enseignant et les formulations des élèves relèvent davantage d'un travail sur des propos sur le monde que sur des objets textuels. Constamment, dans l'exercice qui consiste à apparier au texte la rubrique qui lui correspond, les justifications des uns et des autres portent sur le contenu du texte, et vise à opposer la nature informative vs argumentative des énoncés. Les prises de parole des élèves concernent très accessoirement les conditions de 
l'énonciation qui permettraient de distinguer par exemple le texte de genre " Droit de réponse » dans la rubrique du courrier des lecteurs d'un texte de genre le «Communiqué ». Même si l'ensemble de la construction correspond à une séquence didactique du deuxième groupe, le traitement de la tâche relève davantage d'une préoccupation représentationnaliste de la première famille.

Et enfin, pour la séquence TO13 qui utilise le manuel S'exprimer en français, il est remarquable de constater que l'enseignant enchaîne à la suite d'une activité menée sur des textes de genre, la note critique de lecture, une activité de type repérage sur un texte argumentatif de type dissertation. Sous le titre "J'utilise les mots de liaison », l'activité, manifestement extraite d'un autre manuel, porte sur un texte «à trous"; les mots de liaison ont été supprimés et placés en tête du texte dans le désordre. L'exercice porte autant sur l'enchaînement des phrases que sur l'enchaînement des thèmes à l'intérieur d'une phrase. À ce télescopage de genres correspond un télescopage de thèmes: les notes critiques portent sur des ouvrages de la littérature de jeunesse, tandis que le texte argumentatif porte sur les inconvénients et avantages d'élever des chiots. Ce n'est pas seulement le changement de thématique qui rompt la succession des activités, mais plus fondamentalement le traitement des unités linguistiques dans les exercices menés sur les textes.

Là sans doute, dans ces trois séquences, se dessinent les effets divergents d'une pratique sédimentée.

\section{Remarques conclusives}

La description des suites d'activités rend compte de l'intelligibilité des séquences d'enseignement qui, au-delà des variations singulières liées au style de l'enseignant correspondent à des logiques d'action didactique. Ces logiques échappent pour une part à la variation idiosyncrasique de l'enseignant, et inscrivent celui-ci dans une communauté de pratiques qu'il appartient à la recherche en didactique de spécifier davantage.

La description confirme l'hypothèse de la transposition didactique selon laquelle les pratiques enseignantes sont prises dans des déterminations de différents ordres (didactiques, méthodologiques) reliées aux propriétés des objets enseignés. Cette remarque vaut davantage pour la comparaison de l'ensemble de la collection des séquences SR et TO, portant sur l'objet grammatical et le texte d'opinion.

Une première comparaison de l'ensemble des séquences TO de la collection donne à voir trois grandes familles de suites d'activités. Leur principe unificateur correspond à des préconceptions du langage et du texte ancrées dans des traditions anciennes. La première, de tradition représentationnaliste, considère l'argumentation comme une pratique langagière générale sans spécification de genre. C'est la pensée et les contenus thématiques sur le monde qui prévalent sur les composantes langagières. La deuxième famille, de tradition communicative, entre par les textes de genre, et organise sa progression sur un travail des composantes langagières des textes, neutralisant le plus souvent les contenus. La troisième famille, de tradition philologique ou herméneutique, place au centre de son entreprise le texte littéraire dont elle s'efforce de travailler l'expression en incitant à un retour au texte.

Une description multifocale plus complète devrait permettre une description plus fine de ces épistémologies. Nos premières analyses permettent d'ores et déjà d'observer un phénomène récurrent : celui de la sédimentation des pratiques. En effet, aucune des séquences d'enseignement ne représente une tradition pure; toutes mêlent inextricablement des éléments provenant de traditions différentes. Ces traditions elles-mêmes sont le résultat d'un travail séculaire, voire, millénaire, sur l'enseignement de la production de texte, systématisé dans d'innombrables textes sur la rhétorique, et en particulier son enseignement. Les deux premières familles que nous avons distinguées ont deux manières de puiser dans la rhétorique : l'une, que Ludwig (1988) appelle "'dérhétorisation' de la rhétorique », en garde certains aspects formels tout en faisant abstraction de sa dimension fonctionnelle; l'autre, au contraire, généralise l'entreprise rhétorique en l'intégrant dans le communicatif. Les deux restent cependant incontestablement dans leur découpage de l'objet, la production de textes et son enseignement, héritiers de la rhétorique. A ces deux traditions s'ajoute la troisième, philologique, qui autrement encore, dans l'interprétation, puise dans l'outillage rhétorique. Chacune des traditions ajoute bien sûr des dimensions nouvelles au travail sur les textes. Or, le moment historique auquel nous nous 
trouvons, marqué par une profonde mise en cause des principes fondateurs de la tradition classique et l'irruption massive de la dimension communicative, a pour effet une hybridation particulièrement marquée des pratiques. Le concept de « sédimentation », encore provisoire, essaie de capter cet effet. Il semblerait que les couches les plus solidement ancrées des pratiques, auxquelles spontanément les enseignants ont toujours recours, même dans les pratiques les plus communicatives, soient celles des traditions plus classiques, représentationnelles et philologiques, les autres venant en quelque sorte s'y ajouter. Inversement, les enseignants se situant de manière dominante dans le paradigme classique font, sous des formes diverses, intervenir des éléments de la tradition communicative.

Approfondir, développer, affermir ces premières hypothèses interprétatives plus générales que nous ne pouvons qu'esquisser ici implique un double travail didactique : celui décrit ici, de l'analyse minutieuse, méthodologiquement armée, de la construction de l'objet enseigné dans les pratiques de classe; et celui, tout aussi long et difficile, de l'histoire de la discipline scolaire - magistralement entamée par Chervel (2006) - et de l'histoire des savoirs de référence de la profession enseignante. 


\section{NOTES}

1. Ceci, pour des raisons notamment de l'organisation disciplinaire, est particulièrement vrai pour l'école secondaire, objet de notre étude. Ce n'est pas le lieu ici de discuter la question de cette temporalité et de ses différentes variantes dans le système scolaire.

2. Nous retrouvons ici la revendication affirmée avec force comme l'une des spécificités de la didactique comparée, à savoir que cette dernière « se distingue par l'importance qu'elle attribue à la compréhension fondamentale des pratiques d'enseignement/apprentissage 'telles quelles sont' ce qui suppose une observation des systèmes didactiques pour en dégager leur fonctionnement ordinaire. » (Leutenegger, 2004, p. 3). Pour ce faire, nous prenons résolument comme point de vue l'objet enseigné.

3. Remarquons toutefois la profusion des termes employés, qu'on retrouve aussi bien en classe que dans les plans d'études, 'phrase relative', 'texte argumentatif', etc.

4. Nous sommes conscients, qu'à leur tour, nous l'avons dit, ces objets font partie d'un autre objet de savoir enseigné : la subordonnée relative relève d'un ensemble plus vaste que sont les subordonnées, et ainsi de suite; et que pour comprendre le mode de traitement, il serait nécessaire de connaître la séquence de tous les enseignements. Cela impliquerait un travail longitudinal que nous envisageons de réaliser dans d'autres études.

5. Une première formulation de cette démarche multifocale est exposée dans Schneuwly, B., Cordeiro, G. \& Dolz, J. (2005).

6. Pour une autre tentative de saisir cet objet fuyant la langue première enseignée en classe, utilisant une autre méthodologie, voir Kress Jewitt, Bourne, Franks, Hardcastle, Jones \& Reid (2005); et le livre à paraître de Canelas-Trevisi (sous presse).

7. Pour simplifier le traitement de notre corpus référent, les séquences seront évoquées par une abréviation suivie d'un chiffre, de TO01 à TO17.

8. Une remarque concernant ce groupe de séquences : celles-ci commencent toutes par une production initiale sans que les élèves aient reçu un enseignement systématique qui leur permettrait d'écrire en connaissance de cause. Nous n'avons pas les moyens à ce jour d'établir la corrélation entre cette approche par les genres et cette modalité de traitement du complexe par une première production, ou s'il s'agit seulement d'un rapprochement fortuit. Mais il apparaît nettement que l'articulation de cette activité de production initiale avec l'ensemble de la séquence renvoie à une logique didactique de prise en compte des obstacles des élèves. Lapproche par les genres et la centration sur les conditions de production des textes impliquent à tout le moins une réflexion sur les pratiques sociales, ce qui le rend incompatible avec une démarche ontologique qui se préoccuperait de ne définir que l'objet du monde « argumentation », et cela indépendamment des pratiques argumentatives des élèves.

\section{BIBLIOGRAPHIE}

Brecht, B. (2000/1940). Écrits sur le théâtre. Paris : Gallimard.

Canelas-Trevisi, S. (sous presse). La transposition didactique en français. Analyse des textes pédagogiques et des interactions en classe. Berne : Lang.

Chervel, A. (2006). Histoire de l'enseignement du français du XVII ${ }^{E} a u X X^{E}$ siècles. Paris : Retz.

Chevallard, Y. (1991). La transposition didactique. Grenoble : Éditions La pensée sauvage.

Daunay, B. (2004). Les liens entre écriture d'invention et écriture métatextuelle dans l'histoire de la discipline : quelques interrogations, Skholê, hors série 1, 31-39.

Dolz, J., Noverraz, M., Schneuwly, B. (2001/2002). S'exprimer en français (Vol. I, II, III et IV). Bruxelles : De Boeck-Corome.

Dolz, J., Ronveaux, C. \& Schneuwly, B. (2006). Le synopsis : un outil pour analyser les objets enseignés. In M.J. Perrin-Glorian \& Y. Reuter (Eds), Les méthodes de recherche en didactiques (pp. 175-190). Villeneuve d'Ascq : Presses universitaires du Septentrion. 
Dolz, J., Schneuwly, B., Thévenaz-Christen, T. \& Wirthner, M. (Eds). (2002). Les tâches et leurs entours en classe de français. Conférence introductive du colloque. Actes du 8 e colloque international de la DFLM (Neuchâtel, 26-18 septembre 2001) [CD-ROM]. Neuchâtel : IRDP.

Falardeau, E., Fisher, C., Simard, C. \& Sorin, N. (Eds.). (2007). La didactique du français. Les voies actuelles de la recherche. Québec: Presses de l'Université de Laval.

Hall, R. (2000). Video recording as theory. In D. Lesh \& A. Kelley (Eds.), Handbook of Research Design in Mathematics and Science Education (pp. 647-664). Mahweh, NJ : Lawrence Erlbaum.

Jewitt, C. \& Kress, G. (Eds). (2003). Multimodal literacy. New York : Peter Lang.

Kress, G., Jewitt, C., Bourne, J., Franks, A., Hardcastle, J., Jones, K. \& Reid, E. (2004). English in Urban Classrooms : a multimodal perspective on teaching and learning. London : Routledge Falmer

Leutenegger, F. (2004). Le point de vue de la didactique comparée. Actes du $9^{e}$ Colloque de l'Association Internationale pour la Recherche en Didactique du Français (AIRDF) (Québec, 26 au 26 août 2004). (www. colloqueairdf.fse.ulaval.ca/actes)

Ludwig, O. (1988). Der Schulaufsatz. Seine Geschichte in Deutschland. Berlin : de Gruyter.

Ochs, E. (1979). Transcription as theory. In E. Ochs and B. Schieffelin (Eds.), Developmental pragmatics (pp. 4372, and references 415-429). New York: Academic Press.

Rastier, F. (2001). Arts et sciences du texte. Paris, P. U.F.

Ronveaux, C. \& Simon, A.-C. (en préparation). La gestualité prosodique au service de l'objet enseigné.

Schneuwly, B. (2007). Le « Français» : une discipline scolaire autonome, ouverte et articulée. In E. Falardeau, C. Fisher, C. Simard \& N. Sorin (Eds.), La didactique du français. Les voies actuelles de la recherche (pp. 2035). Québec : Presses de l'Université de Laval.

Schneuwly, B., Cordeiro, G. \& Dolz, J. (2005). À la recherche de l'objet enseigné : une démarche multifocale. Dossiers des sciences de l'éducation, 14, 77-93.

Sensevy, G., Mercier, A., Schubauer-Leoni, M-L., Ligozat, F. \& Perrot, G (2005). An attempt to model the teacher's action in mathematics. Educational Studies in mathematics, 59(1), 153-181.

Stigler, J., Gallimore, R., \& Hiebert, J. (2000). Using video surveys to compare classrooms and teaching across cultures : Examples and lessons from the TIMSS video studies. Educational Psychologist, 35(2), 87-100.

Vygotski, L.S. (1934/1985). Pensée et langage. Paris : Éditions sociales. 
Approches de l'objet enSEIGNÉ...

Christophe Ronveaux \& Bernard Schneuwly

\section{ANNEXES}

\begin{tabular}{|c|c|c|c|c|}
\hline Niveaux & Repères & FST & Matériel & Description \\
\hline 1 & $\begin{array}{l}1 / 6-3 / 6 \\
92 \cdot 30\end{array}$ & & & Définition de l'argumentation \\
\hline $1-1$ & $1 / 657^{\prime}$ & & & Elaboration d'une définition de l'argumentation \\
\hline $1-1-1$ & $1 / 609 ' 48$ & & & > Définir l'argumentation à partir des conceptions des élèves \\
\hline $1-1-2$ & $1 / 610 ’ 37$ & & TN & $>$ Mettre en commun et discuter les propositions faites \\
\hline $1-1-3$ & $1 / 606$ & & & $\begin{array}{l}\text { > Rédiger et critiquer une définition personnelle du terme argumenter à partir des } \\
\text { éléments mis en évidence }\end{array}$ \\
\hline $1-2$ & $1 / 6-2 / 6 ?$ & & & Opérationnalisation de la définition : qu'est-ce qu'une bonne argumentation? \\
\hline $1-2-1$ & $1 / 6-2 / 6 ?$ & $\mathrm{M}$ & & > préparer et réaliser un débat sur le natel \\
\hline \multicolumn{5}{|c|}{ Passage $1 / 6-2 / 6$} \\
\hline $1-2-2$ & $2 / 613 ’ 05$ & & & $\begin{array}{l}\text { > Utiliser la définition de l'argumentation pour décider si une image illustrant une } \\
\text { scène dans laquelle des personnages discutent est une argumentation }\end{array}$ \\
\hline $1-2-3$ & $2 / 614$ & & & $\begin{array}{l}\text { > Mettre en commun les caractéristiques d'une bonne argumentation (orale ou } \\
\text { écrite) }\end{array}$ \\
\hline \multicolumn{5}{|c|}{ Passage $2 / 6-3 / 6$} \\
\hline 2 & $\begin{array}{l}3 / 6-5 / 6 \\
132,50 \\
\end{array}$ & & & Construction d'un modèle de texte argumentatif \\
\hline $2-1$ & $\begin{array}{l}3 / 6-4 / 6 \\
69 \\
\end{array}$ & & $\begin{array}{l}\text { Extrait avec } \\
\text { questions }\end{array}$ & $\begin{array}{l}\text { Lecture et analyse d'un extrait de « Le malade imaginaire » (acte II, scène 3); thème } \\
\text { et arguments. }\end{array}$ \\
\hline $2-1-1$ & $3 / 60620$ & $\mathrm{I}$ & & $>$ Lire le texte et trouver le genre (question 1 ) \\
\hline $2-1-2$ & $3 / 611 ’ 30$ & & & > Repérer le thème et les thèses du dialogue (questions 3 et 4) \\
\hline $2-1-3$ & $3 / 648 ' 00$ & & & $>$ Repérer les arguments \\
\hline \multicolumn{5}{|c|}{ Passage 3/6-6/6 } \\
\hline $2-2$ & $\begin{array}{l}4 / 6-5 / 6 \\
30,55\end{array}$ & & & Synthèse des éléments sur la construction d'une argumentation \\
\hline $2-2-1$ & $4 / 617 ’ 30$ & M, I & $\begin{array}{l}\text { Feuille } \\
\text { brouillon TN }\end{array}$ & $\begin{array}{l}\text { > Noter la structure d'une argumentation (poser le problème, étayer sa théorie } \\
\text { en posant les arguments en sa faveur puis en sa défaveur, respecter l'unité du } \\
\text { paragraphe) }\end{array}$ \\
\hline \multicolumn{5}{|c|}{ Passage 4/6-5/6 } \\
\hline $\begin{array}{l}2-2-1 \\
\text { (suite) }\end{array}$ & $5 / 605 ’ 20$ & I & & $\begin{array}{l}\text { "E" propose aux "és" de terminer le bilan (synthèse) de toutes les informations } \\
\text { sur l'argumentation figurant au TN. Une fois terminé, ce document leur servira de } \\
\text { mémento personnel. }\end{array}$ \\
\hline $2-3$ & $\begin{array}{ll}5 & /\end{array}$ & & & $\begin{array}{l}\text { Lecture et analyse d'un texte d'opinion; les transitions entre parties (Barbara James, } \\
\text { Recycler les déchets, trad. Dominique Boutel, La planète verte, Rageot éditeur, Paris, } \\
\text { 1990). }\end{array}$ \\
\hline $2-3-1$ & $5 / 629 ’ 25$ & $\begin{array}{l}\mathrm{I}+ \\
\mathrm{Q}\end{array}$ & $\begin{array}{l}\text { texte sur le } \\
\text { recyclage }\end{array}$ & $\begin{array}{l}\text { > Retrouver en une phrase l'idée de base de chaque paragraphe du texte sur } \\
\text { le recyclage et signaler toutes les transitions contenues dans ce dernier en les } \\
\text { surlignant. }\end{array}$ \\
\hline \multicolumn{5}{|c|}{ Passage 5/6-6/6 } \\
\hline 3 & $6 / 6-? 37^{\prime} 45$ & & & Production d'un texte argumentatif \\
\hline $3-1$ & $6 / 630 ’ 09$ & & & Exercices d'entraînement \\
\hline $3-1-1$ & $6 / 630 ’ 09$ & & & $>$ Rédiger une introduction \\
\hline $3-2$ & $6 / 67 ’ 45$ & & & Révision de la démarche de production d'un texte argumentatif \\
\hline $3-2-1$ & $6 / 607 ’ 45$ & M & $\begin{array}{l}\text { TN SM } 5 / 6 \\
\text { annexe } 6\end{array}$ & $\begin{array}{l}\text { > Réviser des points importants que les "és" devront respecter lorsqu'ils rédigeront } \\
\text { leur texte argumentatif : le plan }\end{array}$ \\
\hline \multicolumn{5}{|c|}{ Passage 6/6-7 reconstituée } \\
\hline $3-3$ & ? & & & Production d'un texte argumentatif - choix entre trois thèmes \\
\hline
\end{tabular}

Figure 4. Tableau synoptique simplifié de la séquence TO16. 


\begin{tabular}{|c|c|c|c|c|}
\hline 1 & \begin{tabular}{l|l}
$1 / 6-1 / 6$ \\
4343
\end{tabular} & & & Définition de la pétition en contraste avec d'autres genres proches \\
\hline $1-1$ & $\begin{array}{l}1 / 6-1 / 6 \\
846\end{array}$ & Q & & $\begin{array}{l}\text { Définition des caractéristiques de la pétition du point de vue de la situation de } \\
\text { communication }\end{array}$ \\
\hline $1-1-1$ & $1 / 68 ’ 46$ & Q & $\begin{array}{l}\text { Annexe } 1 \\
08.03 .04 \mathrm{au} \text { tn }\end{array}$ & Donner les caractéristiques de la pétition \\
\hline $1-2$ & $\begin{array}{l}1 / 6-1 / 6 \\
31 \cdot 27\end{array}$ & Q & & $\begin{array}{l}\text { Différenciation d'autres genres proches à partir de l'observation de quatre } \\
\text { textes }\end{array}$ \\
\hline $1-2-1$ & $1 / 611 ’ 33$ & Q & $\begin{array}{l}\text { Annexe } 2 \\
08.03 .04 \text { au tn }\end{array}$ & $\begin{array}{l}\text { Examiner, pour des genres proches (lettre de demande, lettre de lecteur, } \\
\text { initiative, pétition), les critères suivants: destinataire, but, énonciateur, } \\
\text { destinataire, genre. }\end{array}$ \\
\hline \multicolumn{5}{|c|}{ Passage leçon 1/6 (08.03.04) à leçon non enregistrée (12.03.04) } \\
\hline 2 & \begin{tabular}{|l|l|l}
$?$ & \\
\end{tabular} & & & Rédaction d'une pétition \\
\hline $2-1-1$ & $?$ & I & & Rédiger une pétition « Pour un cinéclub à l'école» \\
\hline \multicolumn{5}{|c|}{ Passage leçon non enregistrée à 2/6 a et b (19.03.04) } \\
\hline 3 & \begin{tabular}{|l|}
$2 / 6-6 / 6$ \\
$175 ; 30$
\end{tabular} & & & Travail sur les composantes de la pétition \\
\hline $3-1$ & $\begin{array}{l}2 / 6-4 / 6 \\
6454\end{array}$ & & $\begin{array}{l}\text { Annexe } 2 \\
19.03 .04 \mathrm{a} \text { au tn }\end{array}$ & La demande dans son ensemble \\
\hline $3-1-1$ & $2 / 677^{\prime} 01$ & & Fiche 7 1/3 & $\begin{array}{l}\text { Encadrer la demande dans des pétitions et repérer des indices permettant } \\
\text { d'identifier la demande }\end{array}$ \\
\hline $3-1-2$ & $2 / 618$ & I & $\begin{array}{l}\text { Photocopie fiche } \\
81 / 3\end{array}$ & Lire la pétition et souligner les éléments caractéristiques de la demande \\
\hline $3-1-3$ & $2 / 614^{\prime} 06$ & & $\begin{array}{l}\text { Photocopie } \\
\text { séquence fiche } 8\end{array}$ & Compléter une demande lacunaire avec des formules typiques de la demande \\
\hline $3-1-4$ & \begin{tabular}{|l|l|l}
$3 / 515$ & 18 \\
\end{tabular} & $\mathrm{I}$ & Photocopie fiche 9 & Rédiger une demande \\
\hline \multicolumn{5}{|c|}{ Passage leçon 2/6a et b (19.03.04) à 4/6 (23.03.04) } \\
\hline $3-2$ & \begin{tabular}{|l|}
$4 / 6-4 / 6$ \\
$56 \cdot 30$
\end{tabular} & & & La justification \\
\hline $3-2-1$ & $4 / 625^{\prime} 00$ & I & $\begin{array}{l}\text { Photocopie fiche } \\
101 / 1\end{array}$ & Distinguer exemples et arguments et en donner sa définition \\
\hline $3-2-2$ & $4 / 610^{\prime}$ & & $\begin{array}{l}\text { Photocopie } \\
\text { séquence fiche } 11\end{array}$ & $\begin{array}{l}\text { Repérer la partie argumentative d'une pétition lue par un camarade et discutez } \\
\text { de ses bornes; souligner les différents arguments dans le texte }\end{array}$ \\
\hline \multicolumn{5}{|c|}{ Passage leçon 4/6 (23.03.04) à 5/6 a et b (26.03.04) } \\
\hline $3-2-3$ & \begin{tabular}{|l|l|l}
$5 / 616$ \\
\end{tabular} & Q & Textes des élèves & Discuter les arguments produits par les és dans la production initiale \\
\hline $3-3$ & $\begin{array}{l}5 / 6-6 / 6 \\
5406\end{array}$ & & & La mise en forme de la pétition \\
\hline $3-3-1$ & $5 / 622 ’ 30$ & Q & $\begin{array}{l}\text { Annexe } 1 \\
26.03 .04 \mathrm{a} \text { au tn }\end{array}$ & Comparer les procédés de mise en forme de deux pétitions \\
\hline $3-3-2$ & $5 / 62 l^{\prime} 00$ & Q & $\begin{array}{l}\text { Annexe } 2 \\
26.03 .04 \mathrm{a} \text { au tn } \\
\end{array}$ & $\begin{array}{l}\text { Analyser les procédés linguistiques mis en œuvre dans des slogans } \\
\text { publicitaires }\end{array}$ \\
\hline $3-3-3$ & $5 / 610 ’ 36$ & I & $\begin{array}{l}\text { Photocopie fiche } \\
171 / 1\end{array}$ & Mettre en page une pétition \\
\hline \multicolumn{5}{|c|}{ Passage leçon $5 / 6$ a et b à $6 / 6$} \\
\hline 4 & \begin{tabular}{l|l}
$?$ & \\
& 0
\end{tabular} & & & $\begin{array}{l}\text { Production finale : reprise de la production initiale [c'est annoncé dans le } \\
\text { discours de l'enseignante] }\end{array}$ \\
\hline
\end{tabular}

Figure 5. Tableau synoptique de la séquence TO07. 


\begin{tabular}{|c|c|c|c|c|}
\hline 1 & $\begin{array}{l}1 / 9-1 / 9 \\
41^{\prime} 43\end{array}$ & & & $\begin{array}{l}\text { Lecture d'un premier texte argumentatif (la fable de La Fontaine, le Conseil } \\
\text { tenu par les rats [TO05 14.03.03 Annexe 1]) }\end{array}$ \\
\hline $1--1$ & $1 / 9-1 / 98 ' 41$ & & & Lecture et compréhension du texte \\
\hline $1-2$ & $\begin{array}{l}1 / 9-1 / 9 \\
32 \cdot 24\end{array}$ & & & $\begin{array}{l}\text { Analyse de la fable à partir d'un questionnaire (TO05 14.03.03-Annexe 2) : la } \\
\text { structure, les personnages du récit, le débat des rats }\end{array}$ \\
\hline \multicolumn{5}{|c|}{ Passage de la leçon $1 / 9$ à $2 / 9$ (enregistrée en partie, 26') } \\
\hline [1-3] & & $\mathrm{DM}$ & Questionnaire & Ecriture d'invention sur le modèle de la morale de la fable \\
\hline \multicolumn{5}{|c|}{ Passage de la leçon 2/9 à 3/9 } \\
\hline 2 & \begin{tabular}{ll|l}
3 & $/$ & 9 \\
38 & \\
\end{tabular} & & & $\begin{array}{l}\text { Lecture d'un deuxième texte argumentatif (la fable de La Fontaine, La Génisse, } \\
\text { la Chèvre et la Brebis en société avec le Lion [TO05 21.03.03 Annexe 1]) }\end{array}$ \\
\hline $2-1$ & $3 / 95^{\prime} 02$ & & & Lecture et compréhension du texte \\
\hline $2-2$ & $\begin{array}{ll}3 & / \\
32 & 9\end{array}$ & & & $\begin{array}{l}\text { Analyse de la fable à partir d'un questionnaire (TO05 21.03.03 Annexe 1) : la } \\
\text { structure de la fable, l'explicitation du récit et à l'intérieur des dialogues les } \\
\text { arguments du lion }\end{array}$ \\
\hline $2-3$ & 0 '55 & & $\mathrm{DM}$ & Rédaction d'une morale sur le modèle des fables vues \\
\hline \multicolumn{5}{|c|}{ Passage de la leçon 3/9 à 4/9 (reconstituée) } \\
\hline [2-4] & $\begin{array}{l}4 / 9-7 / 9 \\
20,57\end{array}$ & & & $\begin{array}{l}\text { Comparaison de la fable de La Fontaine avec des fables d'Esope, Le Lion, l'Ane et } \\
\text { le Renard, et de Phèdre, Vacca et Capella, Ovis et Leo (TO05 04.04.03 Annexe 1) }\end{array}$ \\
\hline $\begin{array}{l}{[2-4-} \\
1]\end{array}$ & & & & $\begin{array}{l}\text { Lire, comprendre et comparer (cf. le tableau du questionnaire TO05 04.04.03 } \\
\text { Annexe 2) }\end{array}$ \\
\hline \multicolumn{5}{|c|}{ Passage de la leçon 4/9 à 7/9 (supposée) } \\
\hline 3 & $\begin{array}{l}7 / 9-9 / 9 \\
18,56\end{array}$ & & & $\begin{array}{l}\text { Lecture d'un troisième texte argumentatif (la fable de La Fontaine, Le loup et } \\
\text { l'agneau; [TO05 04.04.03 Annexe 4]) }\end{array}$ \\
\hline $3-1$ & $7 / 9-8 / 9$ & & & Lecture et mise en débat de l'analyse de l'argumentation d'une fable \\
\hline 4 & $\begin{array}{l}9 / 9-9 / 9 \\
21 ' ? 544-?\end{array}$ & & & $\begin{array}{l}\text { Rédaction d'une argumentation à travers l'écriture de la suite d'une fable (la } \\
\text { fable de La Fontaine, Le loup et le chien) }\end{array}$ \\
\hline
\end{tabular}

Figure 6. Tableau synoptique de la séquence TO05. 